\title{
ON WEIGHTS WHICH ADMIT THE REPRODUCING KERNEL OF BERGMAN TYPE
}

\section{ZBIGNIEW PASTERNAK-WINIARSKI}

\author{
Institute of Mathematics \\ Technical University of Warsaw \\ Pl. Jedności Robotniczej 1 \\ 00-661 Warsaw, Poland
}

(Received February 7, 1990)

\begin{abstract}
In this paper we consider (1) the weights of integration for which the reproducing kernel of the Bergman type can be defined, i.e., the admissible weights, and (2) the kernels defined by such weights. It is verified that the weighted Bergman kernel has the analogous properties as the classical one. We prove several sufficient conditions and necessary and sufficient conditions for a weight to be an admissible weight. We give also an example of a weight which is not of this class. As a positive example we consider the weight $\mu(z)=(\operatorname{Im} z)^{2}$ defined on the unit disk in $C$.
\end{abstract}

KEY WORDS AND PHRASES. Bergman spaces, Bergman kernel, weighted Bergman function, admissible weights.

1980 AMS SUBJECT CLASSIFICATION CODE. 32HIO.

\section{INTRODUCTION.}

In this paper we are concerned with the weights of integration, the so called admissible weights or a-weights for short for which the Bergman reproducing kernel can be defined. Recently reproducing kernels of Bergman type depending on weights of integration have been used to define the quantization of classical states in holomorphic models of quantum field theory (Odzijewicz [1]). Earlier they have appeared in studies of wave and Dirac equations (Jakobsen and Vergne [2]). Functions of this type have been also considered in consequence of many mathematical problems (Burbea and Masani [3] and Mazur [4]). In (Winiarski [5]) the concept of the a-weight was introduced. The main purpose of the presented study is to give a more detailed characterization of a-weights.

In Section 2 we introduce the new definition of the a-weight. We introduce also the Bergman function defined by such a weight, Definition 2.1 and 2.2. Next we show several basic properties of the Bergman function, Theorem 2.1 and Proposition 2.1. Theorem 2.2 contains some necessary and sufficient conditions for a weight to be an a-weight. Point (iv) of this theorem shows that Definition 2.1 is equivalent to the definition of the a-weight given in [5]. Other more effective sufficient conditions are given in Section 3, Theorem 3.1, and Corollary 3.1 and 3.2. In Section 4 we present the example of a weight which is not an a-weight.

If $\mu$ is a weight, i.e., a real-valued, almost everywhere positive Lebesque measurable function on an open set $D \subset \mathbb{C}^{n}$, then define a set $Z_{\mu}$ as follows: $z \in Z_{\mu}$ iff for any neighborhood $U$ of $z$

$$
\underset{z \in U}{\operatorname{essinf}} \mu(z)=0
$$

All Bergman functions considered to this time have been defined by weights for which $Z_{\mu}=\emptyset$ or the closure of $Z_{\mu}$ is a compact subset of $D$. In Section 5 we deal with the weight $\mu(z)=(\operatorname{Im} z)^{2}$ on the unit disc $D$ in $\mathbb{C}$. In this case $Z_{\mu}=\{z \in D: \operatorname{Im} z=0\}$ is a continuum which "comes to the 
boundary" of $D$. By Corollary $3.2, \mu$ is an a-weight. We find a complete orthonormal system in the space $L^{2} H(D, \mu)$ of all holomorphic $\mu$-square integrable functions on $D$. It makes possible the uniform approximation of the Bergman kernel $K_{\mu}$ by polynomials on any compact subset of $D \times D$, see Theorem 2.1, (i).

Without any other explanations we use the following symbols: $\mathbf{N}$ - the set of natural numbers; $\mathbf{Z}$ - the set of integers; $\mathbf{Z}^{+}:=N \cup\{0\} ; \mathbf{R}$ - the set of reals, $\mathbf{C}$ the complex plane.

\section{ADMISSIBLE WEIGHTS AND REPRODUCING KERNELS.}

Let $D$ be an open nonempty set in $C^{n}$ and let $W(D)$ be the set of weights on $D$, i.e., $W(D)$ is the set of all Lebesque measurable real-valued positive functions on $D$ under the convention that we consider two weights as equivalent if they are equal almost everywhere with respect to the Lebesque measure on $D$. If $\mu \in W(D)$, we denote by $L^{2}(D, \mu)$ the space of all Lebesque measurable complex-valued $\mu$-square integrable functions on $D$.

The space $L^{2}(D, \mu)$ is a separable complex Hilbert space with respect to the norm $\|\cdot\|_{\mu}$ given by the scalar product

$$
<f \mid g>_{\mu}:=\int_{D} \overline{f(z)} g(z) \mu(z)(d z)^{2 n}, \quad f, g \in L^{2}(D, \mu)
$$

The map $L^{2}(D, \mu) \ni f \rightarrow \sqrt{\mu} \cdot f \in L^{2}(D)$ is an isometry on $L^{2}(D, \mu)$ onto the space $L^{2}(D)$ of all complex-valued functions on $D$ which are square integrable with respect to the Lebesque measure. Let $L^{2} H(D, \mu)$ be the set of all holomorphic functions from $L^{2}(D, \mu)$. Then $L^{2} H(D, \mu)$ is a linear subspace of $L^{2}(D, \mu)$ perhaps equal to $\{0\}$ called the $\mu$-Bergman space over $D$ (see [1]). For any $z \in D$ we define the evaluation functional $E_{z}$ on $L^{2} H(D, \mu)$ by the formula

$$
E_{z} f:=f(z), \quad f \in L^{2} H(D, \mu)
$$

DEFINITION 2.1. A weight $\mu \in W(D)$ is called an admissible weight, an a-weight for short, if $L^{2} H(D, \mu)$ is a closed subspace of $L^{2}(D, \mu)$ and for any $z \in D$ the evaluation functional $E_{z}$ is continuous on $L^{2} H(D, \mu)$. The set of all a-weights on D will be denoted by $A W(D)$.

It is well known that the characteristic function of the set $D$ satisfies conditions of the above definition (Krantz [6] and Maurin [7]).

If $\mu \in A W(D)$ then by the Riesz representation theorem, for any $z \in D$ there exists a unique function $e_{z, \mu} \in L^{2} H(D, \mu)$ such that for each $f \in L^{2} H(D, \mu)$

$$
E_{z} f=\left\langle e_{z, \mu} \mid f\right\rangle_{\mu}=\int_{D} \overline{e_{z, \mu}(w)} f(w) \mu(w)(d w)^{2 n}
$$

DEFINITION 2.2. The function $K_{\mu}: D \times D \rightarrow \mathrm{C}$ given by the formula

$$
K_{\mu}(z, w):=\overline{e_{z, \mu}(w)}, \quad z, w \in D
$$

is called the $\mu$-Bergman function of the set $D$ (see [3]). Since the formula (2.2) can be written in the form

$$
f(z)=\int_{D} z_{\mu}(z, w) f(w) \mu(w)(d w)^{2 n}
$$

the function $K_{\mu}$ is also called the $\mu$-Bergman reproducing kernel of $D$.

Similarly as in the classical case we can show the following properties of $K_{\mu}$ (see [3] and [6]). 
THEOREM 2.1. Let $\mu \in A W(D)$ and let $K_{\mu}$ be the $\mu$-Bergman function. Then

(i) for any complete orthonormal system $\left\{\phi_{k}\right\}$ in $L^{2} H(D, \mu)$ and any $(z, w) \in D \times D$

$$
K_{\mu}(z, w)=\sum_{k} \phi_{k}(z) \overline{\phi_{k}(w)}
$$

(ii) for any $(z, w) \in D \times D$

$$
K_{\mu}(w, z)=\overline{K_{\mu}(z, w)}
$$

(iii) the function $K_{\mu}(z, w)$ is antiholomorphic in $w$ and holomorphic in $z$;

(iv) $K_{\mu}$ is analytic in the real sense;

(v) if $P_{\mu}$ is the orthogonal projector on $L^{2}(D, \mu)$ upon $L^{2} H(D, \mu)$ then for every $f \in L^{2}(D, \mu)$ and each $z \in D$

$$
\left[P_{\mu} f\right](z)=\int_{D} K_{\mu}(z, w) f(w) \mu(w)(d w)^{2 n}
$$

i.e., $K_{\mu}$ is the integral kernel of the operator $P_{\mu}$.

PROOF. Let $\left\{\phi_{k}\right\}$ be a complete orthonormal system in $L^{2} H(D, \mu)$ and let for each $z \in D$

$$
\overline{K_{\mu}(z, \cdot)}=e_{z, \mu}=\sum_{k} a_{k}(z) \phi_{k}
$$

where $\left\{a_{k}(z)\right\}$ is the sequence of complex numbers. If $\left\{\phi_{k}\right\}$ is an infinite sequence, the series on the right hand side of $(2.7)$ converges in $L^{2} H(D, \mu)$. Since the evaluation functionals are all continuous we obtain that for every $w \in D$

$$
K_{\mu}(z, w)=\sum_{k} \overline{a_{k}(z)} \overline{\phi_{k}(w)}
$$

On the other hand for any $k$ we have

$$
\phi_{k}(z)=<e_{z, \mu}\left|\phi_{k}>_{\mu}=\sum_{j} \overline{a_{j}(z)}<\phi_{j}\right| \phi_{k}>_{\mu}=\overline{a_{k}(z)}
$$

see (2.2). Applying it to (2.8) we get (2.4). The point (ii) is an immediate consequence of (i).

By its very definition $\overline{K_{\mu}(z, w)}$ is holomorphic in $w$. Hence $K_{\mu}(z, w)$ is antiholomorphic in $w$ and, by (ii), it is holomorphic in $z$.

In order to prove (iv) let us consider the function

$$
D \times D^{0} \ni(z, w) \rightarrow K_{\mu}^{0}(z, w):=K_{\mu}(z, \bar{w}) \in \mathbf{C}
$$

where $D^{0}=\left\{w \in C^{n}: \bar{w} \in D\right\}$. From (iii) it follows that $K_{\mu}^{0}$ is holomorphic in each variable separately. Then by the Hartogs theorem on separate analyticity, it is holomorphic on $D \times D^{0}$ (see [6]).

For the proof of (v) let $f \in L^{2}(D, \mu)$ and let $z \in D$. We have by (i)

$$
\begin{aligned}
& \int_{D} K_{\mu}(z, w) f(w) \mu(w)(d w)^{2 n}=\left\langle e_{z, \mu} \mid f\right\rangle_{\mu} \\
& =<\sum_{k} \overline{\phi_{k}(z)} \phi_{k}\left|f>_{\mu}=\sum_{k} \phi_{k}(z)<\phi_{k}\right| f>_{\mu} \\
& =\left[\sum_{k}<\phi_{k} \mid f>_{\mu} \phi_{k}\right](z)=\left[P_{\mu} f\right](z),
\end{aligned}
$$

where $\left\{\phi_{k}\right\}$ is an arbitrary complete orthonormal system in $L^{2} H(D, \mu)$. This completes the proof of our theorem. 
PROPOSITION 2.1. Under the assumptions of Theorem 2.1. let $\left\{\phi_{m}\right\}$ be an orthonormal complete system in $L^{2} H(D, \mu)$. If $\left\{\phi_{m}\right\}$ is an infinite sequence then the series $\sum_{m=1}^{\infty} \phi_{m}(z) \overline{\phi_{m}(w)}$ converges to $K_{\mu}(z, w)$ uniformly on any compact set $M \subset D \times D$.

PROOF. We can assume that $M=X \times X$, where $X$ is an arbitrary compact subset of $D$, i.e., for any compact set $M \subset D \times D$ there exists a compact set $X \subset D$ such that $M \subset X \times X$. From Theorem 2.1, (i) it follows that for any $z \in D$

$$
\sum_{m=1}^{\infty}\left|\phi_{m}(z)\right|^{2}=K_{\mu}(z, z)<\infty
$$

i.e., $\left(\phi_{m}(z)\right) \in l^{2}$. Let $T_{n}(z):=\sum_{m}^{\infty}\left|\phi_{m}(z)\right|^{2}, z \in D$.

We have that $\left(T_{n}\right)$ is a decreasing sequence of continuous functions on $D$, which converges to $f=0$. By the Dini theorem (Maurin [8]), Chapter V, Section 4) we obtain that $\left(T_{n}\right)$ converges uniformly on any compact set $X \subset D$. The uniform convergence of $\sum_{m=1}^{\infty} \phi_{m}(z) \overline{\phi_{m}(w)}$ on $X \times X$ follows now from the Schwarz inequality

$$
\left|\sum_{m=1}^{\infty} \phi_{m}(z) \overline{\phi_{m}(w)}\right|^{2} \leq T_{n}(z) T_{n}(w), \quad(z, w) \in X \times X .
$$

Now we shall give the necessary and sufficient conditions for a weight $\mu \in W(D)$ to be an aweight.

THEOREM 2.2. Let $\mu \in W(D)$. The following are equivalent:

(i) $\mu$ is an admissible weight;

(ii) for any compact set $X \subset D$ there exists a constant $C_{X}>0$ such that for any $z \in X$ and each $f \in L^{2} H(D, \mu)$

$$
\left|E_{z} f\right| \leq C_{X}\|f\|_{\mu}
$$

(iii) for any $z \in D$ there exists a compact set $Y \subset D$ which contains $z$ and has the following property: for each $z^{\prime} \in \partial Y$ there exists a neighbourhood $V_{z^{\prime}}$ of $z^{\prime}$ in $D$ and a constant $C_{z^{\prime}}>0$ such that for any $w \in V_{z^{\prime}}$ and any $f \in L^{2} H(D, \mu)$

$$
\left|E_{w} f\right| \leq C_{z^{\prime}}\|f\|_{\mu}
$$

$\partial Y$ denotes the topological boundary of $Y$.

(iv) for any $z \in D$ there exists a neighborhood $V_{z}$ of $z$ in $D$ and a constant $C_{z}>0$ such that for any $w \in V_{z}$ and each $f \in L^{2} H(D, \mu)$

$$
\left|E_{w} f\right| \leq C_{z}\|f\|_{\mu} .
$$

PROOF. (i) $\Rightarrow$ (ii). Notice that, by Theorem 2.1, (i) and by the Riesz representation theorem, for any $z \in D$

$$
\left\|E_{z}\right\|_{\mu}=\left\|K_{\mu}(z, \cdot)\right\|_{\mu}=\left(\sum_{m}\left|\phi_{m}(z)\right|^{2}\right)^{1 / 2}=K_{\mu}(z, z)^{1 / 2}
$$

Then, if $X$ is a compact subset of $D$, we have that for any $z \in X$

$$
\left\|E_{z}\right\|_{\mu} \leq C_{X}:=\sup _{w \in X} K_{\mu}(w, w)^{1 / 2}
$$

(ii) $\Rightarrow$ (iii). Let $z \in D$ and let $r>0$ be such that $B(z, 3 r) \subset D$. Set $Y:=\overline{B(z, r)}$ and $X:=\overline{B(z, 2 r)}$. If $C_{X}>0$ is such a constant that (2.10) holds then for any $z^{\prime} \in \partial Y$ we can take $V_{z^{\prime}}:=B\left(z^{\prime}, r\right)$ and $C_{z^{\prime}}:=C_{X}$ 
(iii) $\Rightarrow$ (iv). Let $z \in D$ and let $Y$ be chosen as in the statement (iii) above. If $z \in \partial Y$ then the existence of the neighborhood $V_{z}$ and the constant $C_{z}$ follows immediately form (iii). Suppose now that $z \in \operatorname{int} Y$. Choose for each $z^{\prime} \in \partial Y$ a neighborhood $V_{z^{\prime}}$ and a constant $C_{z^{\prime}}$ such as in (iii). Since $\partial Y$ is compact we can find a finite number of points $z_{1}, z_{2}, \ldots, z_{k} \in \partial Y$ such that

$$
\partial Y \subset \bigcup_{i=1}^{k} V_{z_{i}}
$$

Then for any $z^{\prime} \in \partial Y$ and any $f \in L^{2} H(D, \mu)$

$$
\left|f\left(z^{\prime}\right)\right|=\left|E_{z^{\prime}} f\right| \leq C_{z}\|f\|_{\mu}
$$

where $C_{z}:=\max \left\{C_{z_{1}}, C_{z_{2}}, \ldots, C_{z_{k}}\right\}$. Using now the maximum principle for holomorphic functions we obtain that (2.12) holds for each $w \in V_{z}$ : $=$ int $Y$.

(iv) $\Rightarrow$ (i). The continuity of evaluation functionals follows immediately from (2.12). Moreover, if $\left\{f_{m}\right\}$ is a sequence of elements of $L^{2} H(D, \mu)$ which converges in $L^{2}(D, \mu)$ to a function $f \in L^{2}(D, \mu)$ then, by (iv), this sequence converges locally uniformly on $D$. Hence, by the Weierstrass theorem on the limit of a uniformly convergent sequence of holomorphic functions, the function $f$ is holomorphic, i.e., $f \in L^{2} H(D, \mu)$. This implies that $L^{2} H(D, \mu)$ is a closed subspace of $L^{2}(D, \mu)$.

REMARK. The notion of a $\mu$-Bergman function is not necessary for the proof of the implication (i) $\Rightarrow$ (ii) in the above theorem. One can prove this implication directly from Definition 2.1 using the Banach-Steinhaus theorem on sequences of linear continuous operators (Rudin [9] 5.8).

\section{SUFFICIENT CONDITIONS FOR A WEIGHT TO BE AN ADMISSIBLE WEIGHT.}

A verification of conditions (ii), (iii) or (iv) from Theorem 2.2 usually needs additional considerations and it is sometimes a sufficiently difficult task. In this section we give more effective sufficient conditions for a weight to be an admissible weight.

THEOREM 3.1. Assume that $\mu \in W(D), U$ is an open subset of $D$ and there exists a number $a>0$ such that the function $\mu^{-a}$ is integrable on $U$ with respect to the Lebesque measure. Then for any $z \in U$ there exists a neighborhood $V_{z}$ of $z$ in $D$ and a constant $C_{z}>0$ such that for each $w \in V_{z}$ and each $f \in L^{2} H(D, \mu)$

$$
\left|E_{w} f\right| \leq C_{z}\|f\|_{\mu}
$$

PROOF. If $x^{0} \in \mathbb{C}^{n}, R>0$, define the open ball $B\left(x^{0}, R\right):=\left\{x \in \mathbb{C}^{n}:\left|x-x^{0}\right|<R\right\}$. Let $z \in U$ and let a number $r>0$ be such that $B(z, 2 r) \subset U$. Put $V_{z}:=B(z, r), p:=(1+a) / a$ and $q:=1+a$. We have that, for every $w \in V_{z}, \overline{B(w, r)} \subset U$. (The symbol $\bar{X}$ denotes the closure of the set $X$.) If $f \in L^{2} H(D, \mu)$ then $|f|^{2 / p}$ is a subharmonic function (see [6]), Corollary 2.1 .15 or (Hervé [10] 1.2.2) and therefore

$$
|f(w)|^{\frac{2}{p}} \leq \frac{1}{\operatorname{vol} B(w, r)} \int_{B(w, r)}|f(x)|^{\frac{2}{p}}(d x)^{2 n}, \quad w \in V_{z}
$$

which is the mean value property, (see [6] Theorem 2.14). Since $p, q>1$ and $1 / p+1 / q=1$ we have 
by the Hölder inequality

$$
\begin{aligned}
& \operatorname{vol} B(w, r)|f(w)|^{\frac{2}{p}} \leq \int_{B(w, r)}|f(x)|^{\frac{2}{p}} \mu(x)^{\frac{1}{p}} \mu(x)^{-\frac{1}{p}}(d x)^{2 n} \\
& \leq\left(\int_{B(w, r)}|f(x)|^{2} \mu(x)(d x)^{2 n}\right)^{\frac{1}{p}}\left(\int_{B(w, r)} \mu(x)^{-\frac{q}{p}}(d x)^{2 n}\right)^{\frac{1}{q}} \\
& \leq\|f\|_{\mu}^{2 / p}\left(\int_{U} \mu(x)^{-a}(d x)^{2 n}\right)^{\frac{1}{q}}, \quad w \in V_{z} .
\end{aligned}
$$

Then

$$
|f(w)| \leq\left(\int_{U} \mu(x)^{-a}(d x)^{2 n}\right)^{\frac{p}{2 q}}(\operatorname{vol} B(w, r))^{-\frac{p}{2}}\|f\|_{\mu}
$$

and we can take

$$
C_{z}:=\left(\int_{U} \mu(x)^{-a}(d x)^{2 n}\right)^{\frac{1}{2 a}}(\operatorname{vol} B(w, r))^{-\frac{1+a}{2 a}} .
$$

COROLLARY 3.1. Let $\mu \in W(D)$. Assume that for each $z \in D$ there exists a compact set $Y \subset D$ which contains $z$ and has the following property: for any $w \in \partial Y$ there exists a neighborhood $U_{w}$ of $w$ in $D$ and a number $a_{w}>0$ such that the function $\mu^{-a_{w}}$ is integrable on $U_{w}$ with respect to the Lebesque measure. Then $\mu \in A W(D)$. If in particular, the function $\mu^{-a}$ is locally integrable on $D$ for some $a>0$ then $\mu \in A W(D)$.

PROOF. See Theorems 2.2 and 3.1.

COROLLARY 3.2. Let $\mu \in W(D)$. If for any $z \in D$ there exist a neighborhood $U$ of $z$ in $D$, a constant $a>0$ and a function $f \in C^{1}(U, R)$ such that:

(i) 0 is a regular value of $f$;

(ii) for almost all $w \in U$

$$
\mu(w) \geq|f(w)|^{a}
$$

then $\mu \in A W(D)$.

PROOF. Let $z \in D$ and let $U, a$ and $f$ be associated with $z$ as in the assumptions of the Corollary. If $f(z) \neq 0$ then there exists a bounded neighborhood $U^{\prime}$ of $z$ in $D$ and a constant $b>0$ such that for any $w \in U^{\prime}$

$$
|f(w)|^{a}>b
$$

Hence $\mu(w)^{-1} \leq|f(w)|^{-a}<b^{-1}$, i.e., $\mu^{-1}$ is an integrable function on $U^{\prime}$.

Suppose now that $f(z)=0$. The function $f$ is regular in $z$ which implies that there exists $r>0$ and a bounded diffeomorphism $F: B(0, r) \rightarrow U$ such that $F(0)=z$ and for each $w=\left(w_{1}, \ldots, w_{n}\right) \in B(0, r)$

$$
(f \circ F)(w)=\operatorname{Im} w_{n}
$$

Since $\left|\operatorname{Im} w_{n}\right|^{-\frac{1}{2}}$ is an integrable function on $B(0, r)$ we obtain that $|f|^{-\frac{1}{2}}$ is integrable on $U^{\prime}:=F(B(0, r))$. Hence

$$
\mu^{-\frac{1}{2 a}} \leq|f|^{-\frac{1}{2}}
$$


is an integrable function on $U^{\prime}$. The theorem follows now from Corollary 3.1.

EXAMPLE 3.1. Let $D$ be the unit disk in C. Let for any $z \in D$

$$
\begin{aligned}
f_{t}(z): & =|\operatorname{Im} z|^{t}, \quad t \in(0,+\infty) \\
g(z):= & |\operatorname{Im} z|^{\frac{1}{1-|z|}} ; \\
h(z): & =\left\{\begin{array}{cc}
e^{|z|^{-\frac{1}{2}}} & \text { for } z \neq 0 \\
0 & \text { for } z=0
\end{array}\right.
\end{aligned}
$$

Then the functions $g, h$ and $f_{t}, t \in(0,+\infty)$ are a-weights on $D$.

4. A WEIGHT WHICH IS NOT AN ADMISSIBLE WEIGHT.

In the sequel we shall use the following theorem.

THEOREM 4.1. (Runge). Let $X$ be a compact subset of $\mathbf{C}$ whose complement is connected. Let $f: X \rightarrow C$ be continuous on $X$ and holomorphic on the interior of $X$. Then $f$ is the uniform limit on $X$ of holomorphic polynomials. (See [9], 13.9). Now we are in position to give the example of the weight which is not an a-weight. Let $D:=\{z \in \mathrm{C}:|z|<1\}$ be the unit disk in C. Let $n \in \mathbb{N}$. Define

$$
\begin{gathered}
A_{n}:=B\left(0,2^{-n}\right) \cup\left\{z \in D: \operatorname{Re} z>0,|\operatorname{Im} z|<2^{-n}\right\}, \\
M_{n}:=\left(\bar{D}-A_{n}\right) \cup \bar{A}_{n+1}
\end{gathered}
$$

where $\bar{D}$ and $\bar{A}_{n+1}$ denotes the closure in $\mathrm{C}$ of $D$ and $A_{n+1}$ respectively. Let $f_{n}: M_{n} \rightarrow \mathrm{C}$ be given the formula

$$
f_{n}(z):= \begin{cases}1+\frac{1}{n} & \text { for } z \in \bar{A}_{n+1} \\ 0 & \text { for } z \in \bar{D}-A_{n}\end{cases}
$$

Since the set $\mathrm{C}-M_{n}$ is connected we obtain by the Runge theorem that there exists a holomorphic polynomials $g_{n}$ such that for any $z \in M_{n}$

$$
\left|f_{n}(z)-g_{n}(z)\right|<1 / n
$$

We have that $\left|g_{n}(z)\right|<1 / n$ for $z \in \bar{D}-A_{n}$ and $1<\left|g_{n}(z)\right|<1+2 / n$ for $z \in \bar{A}_{n+1}$. Let

$$
h_{n}(z):=\frac{1}{g_{n}(0)} g_{n}(z), \quad z \in D .
$$

Since $\left|g_{n}(0)\right|>1$ we have that $\left|h_{n}(z)\right|<1 / n$ for $z \in D-A_{n}$ and $\left|h_{n}(z)\right|<1+2 / n$ for $z \in \bar{A}_{n+1} \cap D$. Moreover, $h_{n}(0)=1$.

Now define a weight $\mu \in W(D)$ as follows:

$$
\mu(z):= \begin{cases}1 & \text { for } z \in D-A_{1} \\ 0 & \text { for } z \in[0,1) \subset R \\ \min _{n \in \mathbb{N}}\left\{1,1 /\left|h_{n}(z)\right|^{2}\right\} & \text { for } z \in A_{n}-A_{n+1}\end{cases}
$$

We have that for any $z \in D, \mu(z) \leq 1$. It is clear that $h_{n} \in L^{2} H(D, \mu)$ for $n \in \mathbb{N}$ and $E_{0} h_{n}=1$. 
Notice that for each $z \in D$

$$
\left|h_{n}(z)\right|^{2} \mu(z)<9 \text { and } \lim _{n \rightarrow \infty}\left|h_{n}(z)\right|^{2} \mu(z)=0
$$

Then by the Lebesque theorem on majorized convergence

$$
\lim _{n \rightarrow \infty}\left\|h_{n}\right\|_{\mu}^{2}=\lim _{n \rightarrow \infty} \int_{D}\left|h_{n}(z)\right|^{2} \mu(z)(d z)^{2 n}=0
$$

whereas $\lim _{n \rightarrow \infty} E_{0} h_{n}=1$. This implies that the evaluation functional $E_{0}$ is not continuous on $L^{2} H(D, \mu)$ and therefore $\mu$ is not an admissible weight on $D$.

5. THE WEIGHT $\mu(z)=(\operatorname{Im} z)^{2}$ ON THE UNIT DISC IN C.

We are going to find a complete orthonormal system in $L^{2} H(D, \mu)$. Let for any $n \in Z^{+}$

$$
g_{n}(z):=z^{n}, \quad z \in D
$$

If $r>0$ define $D r:=\{z \in C:|z|<r\}$. Since

$$
(\operatorname{Im} z)^{2}=\frac{1}{2}|z|^{2}-\frac{1}{4}\left(z^{2}+\bar{z}^{2}\right)
$$

one can easy verify that

$$
\int_{D r} \overline{g_{m}(z)} g_{k}(z) \mu(z) d^{2} z:=\left\{\begin{aligned}
& \frac{\pi r^{2 m+4}}{2(m+2)} \text { for } k=m, \\
&-\frac{\pi r^{2 m+2}}{4(m+1)} \text { for } k=m-2, \\
&-\frac{\pi r^{2 m+6}}{4(m+3)} \text { for } k=m+2, \\
& 0 \text { in the other cases. }
\end{aligned}\right.
$$

Setting $r=1$ we obtain

$$
<g_{m} \mid g_{k}>_{\mu}:=\left\{\begin{array}{cl}
\frac{\pi}{2(m+2)} & \text { for } k=m, \\
-\frac{\pi}{4(m+1)} & \text { for } k=m-2, \\
-\frac{\pi}{4(m+3)} & \text { for } k=m+2, \\
0 \text { in the other cases. }
\end{array}\right.
$$

Notice that for any $k, l \in \mathbf{Z}^{+}$

$$
<g_{2 k}\left|g_{2 l+1}\right\rangle_{\mu=0}
$$

This implies that there exists such an orthogonal system $\left(f_{n}\right)_{n=0}^{\infty}$ in $L^{2} H(D, \mu)$ that for any $j \in \mathbf{Z}^{+}$

$$
\left\{\begin{array}{l}
f_{2 j} \in \operatorname{Span}\left\{g_{0}, g_{2}, \ldots, g_{2 j}\right\} \\
f_{2 j+1} \in \operatorname{Span}\left\{g_{1}, g_{3}, \ldots, g_{2 j+1}\right\}
\end{array}\right.
$$


(In particular the sequence obtained from $\left(g_{n}\right)$ by the Schmidts orthonormalization procedure has the above property.) Let $\left(f_{n}\right)$ be such a system. We have

$$
\left\{\begin{array}{l}
f_{2 j}=\sum_{k=0}^{j} a_{2 j, 2 k} g_{2 k}, \\
f_{2 j+1}=\sum_{k=0}^{j} a_{2 j+1,2 k+1} g_{2 k+1},
\end{array} \quad j \in \mathbf{Z}^{+}\right.
$$

and

$$
<f_{n} \mid g_{m}>_{\mu}=0
$$

for each $0 \leq m<n, n \in \mathbb{N}$. By (5.2), (5.4) and (5.5) we get

$$
0=<g_{m} \mid \sum_{p=0}^{n} a_{n, p} g_{p}>_{\mu}=-\frac{\pi}{4(m+1)} a_{n, m-2}+\frac{\pi}{2(m+2)} a_{n, m}-\frac{\pi}{4(m+3)} a_{n, m+2}
$$

for $0 \leq m<n$. Then we have the following recurrent formulas

$$
\left\{\begin{array}{l}
a_{2 j, 2}=3 a_{2 j, 0}, a_{2 j+1,3}=\frac{8}{3} a_{2 j+1,1}, \quad j \in Z^{+} \\
a_{n, m+2}=2 \frac{m+3}{m+2} a_{n, m}-\frac{m+3}{m+1} a_{n, m-2} \text { for } m>2, \quad n \in Z^{+}
\end{array}\right.
$$

where $a_{2 j, 0}$ and $a_{2 j+1,1}$ are arbitrary complex numbers. Notice that, by (5.4), $a_{n, m}=0$ for $n-m$ to be an odd number. By (5.6) it is clear that $a_{2 j, 2 k}$ is a linear function of the variable $a_{2 j, 0}$, whereas $a_{2 j+1,2 k+1}$ is a linear function of $a_{2 j+1,1}$ for $k \leq j$.

From now on we fix $\left(f_{n}\right)$ taking

$$
a_{2 j, 0}=a_{2 j+1,1}=1
$$

for each $j \in \mathbf{Z}^{+}$. If $i, j, k \in \mathbf{Z}^{+}$and $k \leq \min \{i, j\}$ then

$$
a_{2 j, 2 k}=a_{2 i, 2 k} \text { and } a_{2 j+1,2 k+1}=a_{2 i+1,2 k+1}
$$

Therefore we shall write $a_{k}$ instead of $a_{n, k}$ for each $n, k \in \mathbf{Z}^{+}, k \leq n$. We have

$$
\begin{gathered}
f_{2 j}=\sum_{k=0}^{j} a_{2 k} g_{2 k} \\
f_{2 j+1}=\sum_{k=0}^{j} a_{2 k+1} g_{2 k+1}, \quad j \in \mathbf{Z}^{+},
\end{gathered}
$$

where

$$
\begin{gathered}
a_{0}=1, a_{1}=1, a_{2}=3, a_{3}=\frac{3}{8} \\
a_{m+2}=2 \frac{m+3}{m+2} a_{m}-\frac{m+3}{m+1} a_{m-2} \text { for } m \geq 2
\end{gathered}
$$

It is obvious that all $a_{k}$ are real numbers. 
Let us calculate the norm $\|f\|_{\mu}$. By (5.2) and (5.8) we get

$$
\begin{aligned}
\left\|f_{0}\right\|_{\mu}^{2} & =\frac{\pi}{4},\left\|f_{1}\right\|_{\mu}^{2}=\frac{\pi}{6} \\
\left\|f_{m}\right\|_{\mu}^{2} & =<f_{m}\left|f_{m-2}+a_{m} g_{m}>_{\mu}=a_{m}<f_{m}\right| g_{m}>\mu= \\
& =a_{m}\left(-\frac{\pi}{4(m+1)} a_{m-2}+\frac{\pi}{2(m+2)} a_{m}\right) \\
& =\frac{\pi}{4(m+3)} a_{m} a_{m+2}, \quad \text { for } m \geq 2 .
\end{aligned}
$$

Since $f_{m} \neq 0$ we obtain that $a_{m} \neq 0$ for each $m \in \mathbf{Z}^{+}$. Now define a sequence $\left(\phi_{m}\right)$ of elements of $L^{2} H(D, \mu)$ by the formula:

$$
\phi_{m}:=\frac{1}{\left\|f_{m}\right\|_{\mu}} f_{m}=2\left(\frac{m+3}{\pi a_{m} a_{m+2}}\right)^{\frac{1}{2}} f_{m} .
$$

We are going to show that the orthonormal system $\left(\phi_{m}\right)$ is complete in $L^{2} H(D, \mu)$.

LEMMA 5.1. If $\left(a_{m}\right)$ is a sequence defined by (5.8) then for any $m \in \mathbb{N}$

$$
\left(1+\frac{3}{m+2}\right) a_{m}<a_{m+2}<\left(1+\frac{4}{m}\right) a_{m}
$$

PROOF. By a direct calculation we can verify (5.11) for $m=1$ and $m=2$. Suppose now that it is true for each $m \leq n-1$, where $n \in \mathbb{N}$ and $n>1$. In particular

$$
\left(1+\frac{3}{n}\right) a_{n-2}<a_{n}=a_{(n-2)+2}<\left(1+\frac{4}{n-2}\right) a_{n-2} .
$$

Then

$$
a_{n-2}<\frac{n}{n+3} a_{n}
$$

and

$$
a_{n+2}=\frac{2 n+6}{n+2} a_{n}-\frac{n+3}{n+1} a_{n-2}>\left(\frac{2 n+6}{n+2}-\frac{n}{n+1}\right) a_{n}=\frac{n^{2}+6 n+6}{(n+1)(n+2)} a_{n}
$$

Since

$$
\frac{n^{2}+6 n+6}{(n+1)(n+2)}>\frac{n+5}{n+2}
$$

we get

$$
a_{n+2}>\frac{n+5}{n+2} a_{n}=\left(1+\frac{3}{n+2}\right) a_{n}
$$

From (5.12) it follows that

$$
a_{n-2}>\frac{n-2}{n+2} a_{n}
$$

Hence

$$
\begin{gathered}
a_{n+2}=\frac{2 n+6}{n+2} a_{n}-\frac{n+3}{n+1} a_{n-2} \\
<\left(\frac{2 n+6}{n+2}-\frac{n+3}{n+1} \frac{n-2}{n+2}\right) a_{n}=\frac{n^{2}+7 n+12}{(n+1)(n+2)} a_{n} .
\end{gathered}
$$


Since

$$
\frac{n+4}{n}-\frac{n^{2}+7 n+12}{(n+1)(n+2)}=\frac{2 n+8}{n(n+1)(n+2)}>0
$$

we obtain

$$
a_{n+2}<\frac{n+4}{n} a_{n}=\left(1+\frac{4}{n}\right) a_{n} .
$$

Finally we can state that (5.11) is true for $m=n$ and by the induction principle it is true for any $m \in \mathbb{N}$.

COROLLARY 5.1. The series $\sum_{n=0}^{\infty} a_{2 j} z^{2 j}$ and $\sum_{j=0}^{\infty} a_{2 j+1} z^{2 j+1}$ converge on $D$.

PROOF. By (5.11) we get $\lim _{j \rightarrow \infty} \frac{a_{2 j+2}}{a_{2 j}}=\lim _{j \rightarrow \infty} \frac{a_{2 j+3}}{a_{2 j+1}}=1$.

Hence, by d'Alembert's test, the series

$$
\sum_{j=0}^{\infty} a_{2 j} w^{j} \text { and } \sum_{j=0}^{\infty} a_{2 j+1} w^{j} \text { converge for }|w|<1
$$
Taking $w=z^{2}$ we obtain that the series $\sum_{j=0}^{\infty} a_{2 j} z^{2 j}$ and $\sum_{j=0}^{\infty} a_{2 j+1} z^{2 j+1}=z \cdot \sum_{j=0}^{\infty} a_{2 j+1} z^{2 j}$
converge on $D$.

LEMMA 5.2. Let $\left(a_{n}\right)$ be a sequence defined by the formula (5.8). Then for any $j \in \mathbf{N}$

$$
\begin{gathered}
a_{2 j}^{2}>\frac{(2 j+3)(j+2)(j+1)}{6} ; \\
a_{2 j+1}^{2}>\frac{(2 j+5)(2 j+3)(j+2)}{30} .
\end{gathered}
$$

PROOF. Using $j$ times the inequality (5.11) we have

Let

$$
a_{2 j}>\prod_{k=1}^{j}\left(1+\frac{3}{2 k}\right), a_{2 j+1}>\prod_{k=1}^{j}\left(1+\frac{3}{2 k+1}\right)
$$

$$
A_{2 j}:=\prod_{k=1}^{j}\left(1+\frac{3}{2 k}\right), A_{2 j+1}:=\prod_{k=1}^{j}\left(1+\frac{3}{2 k+1}\right)
$$

It is clear that $A_{2 j}>A_{2 j+1}$. Hence

$$
\begin{aligned}
A_{2 j}^{2}>A_{2 j} A_{2 j+1} & =\frac{2 j+4}{2 j+1} \frac{2 j+3}{2 j} \frac{2 j+2}{2 j-1} \frac{2 j+1}{2 j-2} \cdots \frac{8}{5} \frac{7}{4} \frac{6}{3} \frac{5}{2} \\
& =\frac{(2 j+4)(2 j+3)(2 j+2)}{4 \cdot 3 \cdot 2}
\end{aligned}
$$

which implies (5.13). We have also

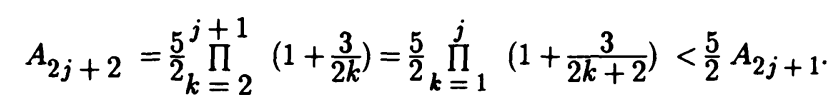

Then

which implies (5.14).

$$
\begin{aligned}
\frac{5}{2} A_{2 j+1}^{2}>A_{2 j+2} A_{2 j+1} & =\frac{2 j+5}{2 j+2} \frac{2 j+4}{2 j+1} \frac{2 j+3}{2 j} \frac{2 j+2}{2 j-1} \cdots \frac{8}{5} \frac{7}{4} \frac{6}{3} \frac{5}{2} \\
& =\frac{(2 j+5)(2 j+4)(2 j+3)}{4 \cdot 3 \cdot 2}
\end{aligned}
$$

THEOREM 5.1. The system $\left(\phi_{m}\right)$ given by the formula (5.10) is complete in $L^{2} H(D, \mu)$.

PROOF. Let $f(z)=\sum_{k=0}^{\infty} b_{k} z^{k}$ be an element of $L^{2} H(D, \mu)$ which is orthogonal to any $\phi_{m}$, 
$m \in \mathbf{Z}^{+}$. Since for each $m \in \mathbf{Z}^{+} g_{m} \in \operatorname{Span}\left\{\phi_{1} \phi_{2}, \ldots\right\}$ we have $\left\langle f \mid g_{m}\right\rangle_{\mu}=0$. On the other hand, for any $0<r<1$ the series $\sum_{k=0}^{\infty} b_{k} z^{k}$ converges to $f(z)$ uniformly on the disk Dr.

Therefore, by (5.1)

$$
\int_{\mathrm{Dr}} \overline{g_{0}(z)} f(z) \mu(z) d^{2} z=\sum_{k=0}^{\infty} b_{k} \int_{\mathrm{Dr}} z^{k} \mu(z) d^{2} z=\frac{\pi r^{4}}{4} b_{0}-\frac{\pi r^{6}}{12} b_{2}
$$

Similarly

$$
\int_{\mathrm{Dr}} \overline{g_{1}(z)} f(z) \mu(z) d^{2} z=\frac{\pi r^{6}}{6} b_{1}-\frac{\pi r^{8}}{16} b_{3}
$$

and

$$
\int_{\mathrm{Dr}} \overline{g_{m}(z)} f(z) \mu(z) d^{2} z=-\frac{\pi r^{2 m+2}}{4(m+1)} b_{m-2}+\frac{\pi r^{2 m+4}}{2(m+2)}-\frac{\pi r^{2 m+6}}{4(m+3)}, \quad \text { for } m>1 .
$$

If $r \rightarrow 1$ we obtain equations which are identical with the equations (5.6) in which $a_{n, m}$ are exchanged for $b_{m}, m \in \mathbf{Z}^{+}$. This implies that for any $j \in \mathbf{Z}^{+}$

$$
b_{2 j}=a_{2 j} b_{0} \text { and } b_{2 j+1}=a_{2 j+1} b_{1}, \quad b_{0}, b_{1} \in \mathbf{C}
$$

where $\left(a_{m}\right)$ is the sequence defined by (5.8).

Let $F_{0}(z)=\sum_{j=0}^{\infty} a_{2 j} z^{2 j}$ and $F_{1}(z)=\sum_{j=0}^{\infty} a_{2 j+1} z^{2 j+1}, z \in D$. From Corollary 5.1 it follows that $F_{1}$ and $F_{2}$ are well defined holomorphic functions on D. We have $f=b_{0} F_{0}+b_{1} F_{1}$. Since by $(5.1)$

$$
\int_{\mathrm{Dr}} \overline{F_{0}(z)} F_{1}(z) \mu(z) d^{2} z=\sum_{j, k=0}^{\infty} a_{2 j} a_{2 k+1} \int_{\mathrm{Dr}} \overline{g_{2 j}(z)} g_{2 k+1}(z) \mu(z) d^{2} z=0
$$

we obtain that

$$
\int_{\mathrm{Dr}}|f(z)|^{2} \mu(z) d^{2} z=\left|b_{0}\right|^{2} \int_{\mathrm{Dr}}\left|F_{0}(z)\right|^{2} \mu(z) d^{2} z+\left|b_{1}\right|^{2} \int_{\mathrm{Dr}}\left|F_{1}(z)\right|^{2} \mu(z) d^{2} z
$$

Hence

$\|f\|_{\mu}^{2}=\lim _{r \rightarrow 1} \int_{\mathrm{Dr}}|f(z)|^{2} \mu(z) d^{2} z=\lim _{r \rightarrow 1}\left[\left|b_{0}\right|^{2} \cdot \int_{\mathrm{Dr}}\left|F_{0}(z)\right|^{2} \mu(z) d^{2} z+\left|b_{1}\right|^{2} \int_{\mathrm{Dr}}\left|F_{1}(z)\right|^{2} \mu(z) d^{2} z\right]$

Notice that

$$
\begin{aligned}
& \left.\left.\int_{\mathrm{Dr}}\right|_{k=0} \sum_{k=0}^{n} a_{2 k} z^{2 k}\right|^{2} \mu(z) d^{2} z=\sum_{k, m=0}^{n} a_{2 k} a_{2 m} \int_{\mathrm{Dr}} \overline{g_{2 k}(z)} g_{2 m}(z) \mu(z) d^{2} z \\
& =\sum_{m=0}^{n} \frac{\pi r^{4 m+4}}{2(2 m+2)} a_{2 m}^{2}-\sum_{m=1}^{n} \frac{\pi r^{4 m+2}}{4(2 m+1)} a_{2 m-2} a_{2 m}-\sum_{m=0}^{n-1} \frac{\pi r^{4 m+6}}{4(2 m+3)} a_{2 m} a_{2 m+2}
\end{aligned}
$$


After further calculations we get

$$
\int_{\mathrm{Dr}}\left|\sum_{k=0}^{n} a_{2 k} z^{2 k}\right|^{2} \mu(z) d^{2} z=\frac{\pi r^{4 n+4}}{4} \frac{a_{2 n} a_{2 n+2}}{2 n+3}+\frac{\pi\left(1-r^{2}\right)^{2}}{4} \sum_{k=0}^{n-1} r^{4 k+4} \frac{a_{2 k} a_{2 k+2}}{2 k+3}
$$

Using Lemma 5.1 and 5.2 we see that

$$
\lim _{n \rightarrow \infty} \frac{\pi}{4} r^{4 n+4} \frac{a_{2 n} a_{2 n+1}}{2 n+3}=0
$$

and

$$
\begin{aligned}
& \int_{\mathrm{Dr}}\left|F_{0}(z)\right|^{2} \mu(z) d^{2} z=\frac{\pi\left(1-r^{2}\right)^{2}}{4} \sum_{k=0}^{\infty} r^{4 k+4} \frac{a_{2 k} a_{2 k+2}}{2 k+3} \\
& >\frac{\pi\left(1-r^{2}\right)^{2}}{4} \cdot \sum_{k=0}^{\infty} r^{4 k+4} a_{2 k}^{2} \frac{2 k+5}{(2 k+2)(2 k+3)} \\
& >\frac{5 \pi r^{4}\left(1-r^{2}\right)^{2}}{24}+\frac{\pi\left(1-r^{2}\right)^{2}}{4} \sum_{k=1}^{\infty} \frac{(2 k+5)(2 k+3)(k+2)(k+1)}{12(k+1)(2 k+3)} r^{4 k+4} \\
& =\frac{\pi\left(1-r^{2}\right)^{2}}{48} \sum_{k=0}^{\infty}(2 k+5)(k+2) r^{4 k+4}
\end{aligned}
$$

Let $s:=r^{4}$. Since $\frac{2 k+5}{k+3}>\frac{5}{3}$ we have

$$
\sum_{k=0}^{\infty}(2 k+5)(k+2) r^{4 k+4}>\frac{5}{3} \sum_{k=0}^{\infty}(k+3)(k+2) s^{k+1}=\frac{5}{3} \frac{2 s^{3}-6 s^{2}+6 s}{(1-s)^{3}}=\frac{5}{3} r^{4} \frac{2 r^{8}-6 r^{4}+6}{\left(1-r^{2}\right)^{3}\left(1+r^{2}\right)^{3}}
$$

Hence

$$
\int_{\mathrm{Dr}}\left|F_{0}(z)\right|^{2} \mu(z) d^{2} z>\frac{5 \pi}{72} r^{4} \frac{r^{8}-3 r^{4}+3}{\left(1-r^{2}\right)\left(1+r^{2}\right)^{3}}
$$

and as a consequence

$$
\lim _{\substack{r \rightarrow 1 \\ r<1}} \int_{\operatorname{Dr}}\left|F_{0}(z)\right|^{2} \mu(z) d^{2} z=+\infty
$$

Analogously, one can show that for any $0<r<1$

$$
\int_{\operatorname{Dr}}\left|F_{1}(z)\right|^{2} \mu(z) d^{2} z>\frac{\pi}{30} \frac{r^{12}-6 r^{8}+6 r^{4}}{\left(1-r^{2}\right)\left(1+r^{2}\right)^{3}}
$$

and therefore

$$
\lim _{\substack{r \rightarrow 1 \\ r<1}} \int_{\mathrm{Dr}}\left|F_{1}(z)\right|^{2} \mu(z) d^{2} z=+\infty
$$

Formulas (5.17), (5.18), and (5.19) imply that $b_{0}=b_{1}=0$ and as a consequence $f=0$. This completes the proof of our theorem. 
ACKNOWLEDGEMENT. The author would like to express his gratitude to Professor Maciej Skwarczyński. His suggestions and support through the development of the paper were greatly appreciated.

Special thanks are due to my wife for her help.

\section{REFERENCES}

1. ODZIJEWICZ, A. On Reproducing Kernels and Quantization of States, Commun. Math. Phys. 114, (1988), 577-597.

2. JAKOBSEN, H.P. and VERGNE, M. Wave and Dirac Operators and Representations of the Conformal Group, J. Funct. Anal. 24, (1977), 52-106.

3. BURBEA, J. and MASANI, P. Banach and Hilbert Spaces of Vector-Valued Functions, Research Notes in Mathematics 90, Pitman, Boston, 1984.

4. MAZUR, T. Canonical Isometry on Weighted Bergman Spaces, Pac. J. Math. 136(2), (1989), 303-310.

5. PASTERNAK-WINIARSKI, Z. On the Dependence of the Reproducing Kernel on the Weight of Integration, J. Funct. Anal. 94. (1990), 110-134.

6. KRANTZ, S.G. Function Theory of Several Complex Variables, Intersciences-Wiley, New York, 1982.

7. MAURIN, K. Analysis, Part II. Integration, Distributions, Holomorphic Functions, Tensor and Harmonic Analysis, PWN-Reidel, Warszawa-Dordrecht, Boston, London, 1980.

8. MAURIN, K. Analysis, Part I. Elements, PWN-Reidel, Warszawa-Dordrecht, Boston, London, 1976.

9. RUDIN, W. Real and Complex Analysis, McGraw-Hill Inc., 1974.

10. HERVÉ, M. Analytic and Plurisubharmonic Functions in Finite and Infinite Dimensional Spaces, Lecture Notes in Mathematics 198, Springer-Verlag, Berlin, 1971. 


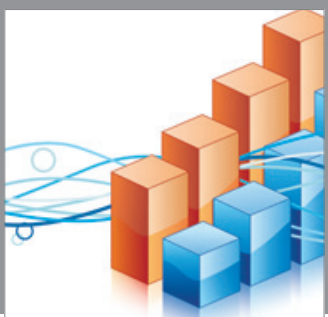

Advances in

Operations Research

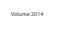

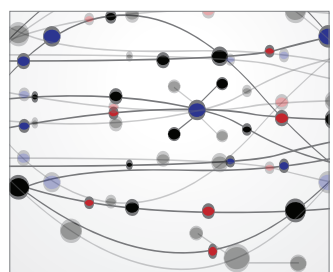

\section{The Scientific} World Journal
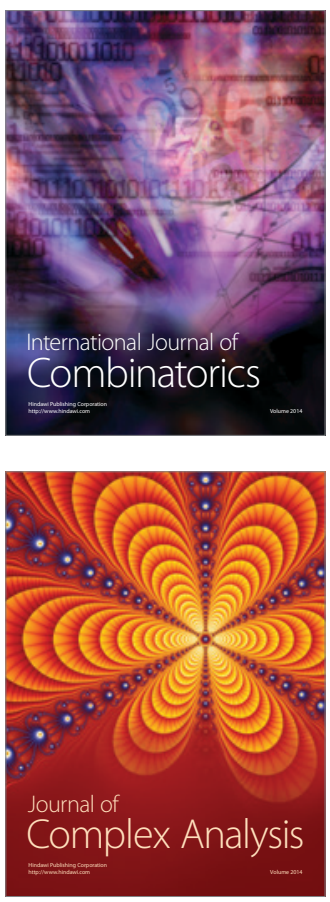

International Journal of

Mathematics and

Mathematical

Sciences
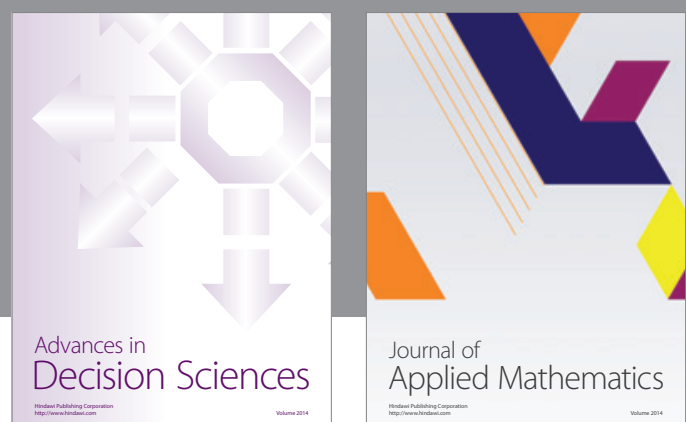

Journal of

Applied Mathematics
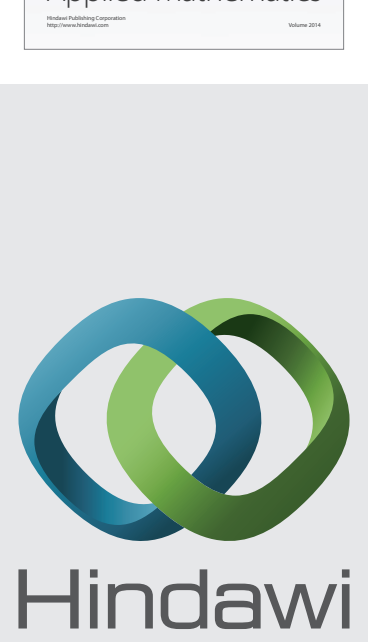

Submit your manuscripts at http://www.hindawi.com
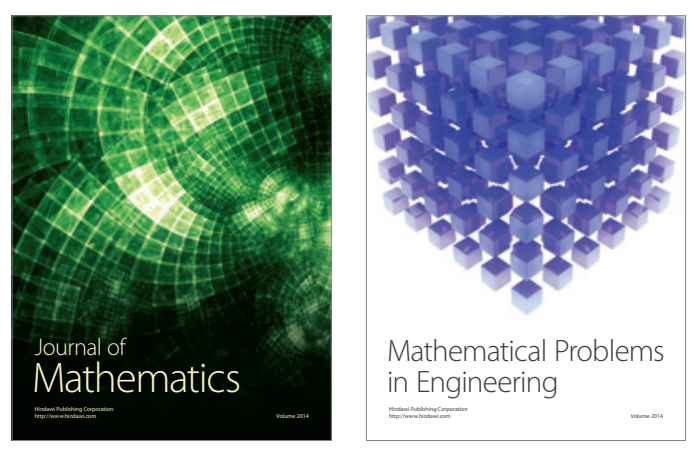

Mathematical Problems in Engineering
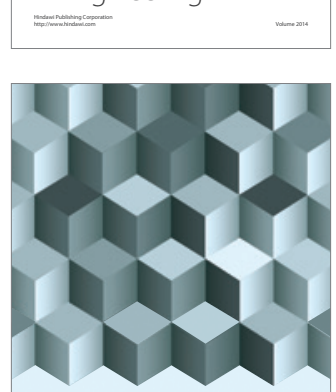

Journal of

Function Spaces
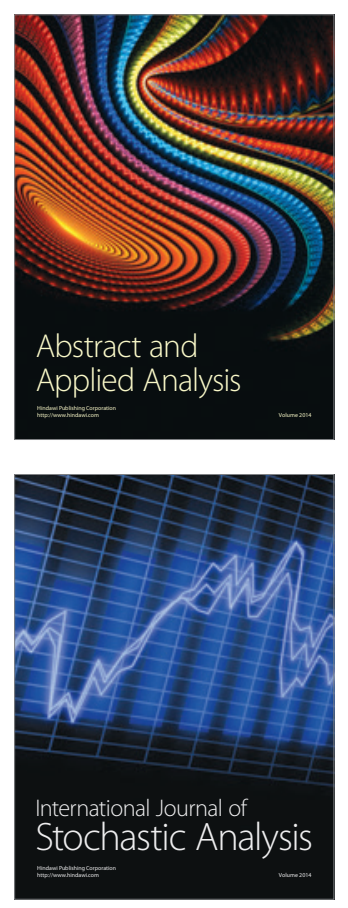

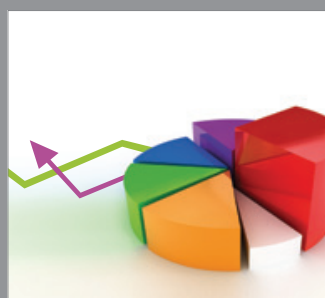

ournal of

Probability and Statistics

Promensencen
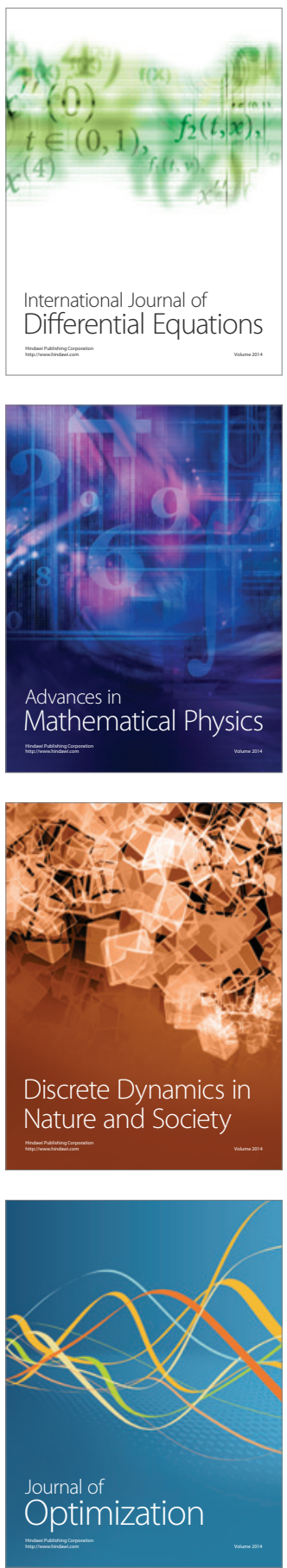